\title{
3D Printing Pen: A Novel Adjunct for Indirect Bonding
}

\author{
Narayan Bhalchandra Kulkarni ${ }^{1}$, Shrishtee Manoj Goyal ${ }^{2}$, Vinaykumar Dinesh Shah ${ }^{3}$
}

\begin{abstract}
Background: Direct orthodontic bracket bonding consumes significantly more chairside time in comparison to indirect bonding. The primary drawback of indirect bonding is incomplete penetration of the curing light through transfer trays, leading to bracket bond failure. The primary objective in a clinical setting is to minimize the chairside time. This technique of indirect bonding reduces chairside time, is economical, utilizes minimum armamentarium, and provides adequate light penetration and accuracy.

Technique: Impressions were made, casts were poured and marked for the desired bracket position as per MBT prescription. Bonding agent was used to stabilize the brackets on the working model. A 3D printing pen and polylactic acid (PLA) filament were used to fabricate transfer trays for indirect bonding. The tray with brackets held in the desired position was transferred to the oral cavity and cured.

Conclusions: Fabrication of complete or partial transfer trays for indirect bonding using PLA with the help of a 3D printing pen is a precise, easy-to-use, economical, and reliable method that reduces chairside time.

Clinical significance: When compared with other methods of indirect bonding, the PLA transfer tray made with a 3D printing pen was easy to fabricate with brackets held firmly in the tray for a precisely desired position. The tray fit snugly and permitted light curing of composite from all surfaces of brackets and removal of flash. This method significantly reduced the overall time of the indirect bonding procedure.

Keywords: 3D printing, Bonding, Indirect bonding, Polylactic acid (PLA).

The Journal of Contemporary Dental Practice (2021): 10.5005/jp-journals-10024-3166
\end{abstract}

\section{BACKGROUND}

Indirect orthodontic bonding was first introduced in 1972 by Silverman and Cohen; ${ }^{1}$ brackets were positioned and stuck on the working model and a transfer tray was constructed for the placement of brackets in the oral cavity. Schwartz in $1974^{2}$ introduced the sugar daddy technique which advocated the use of caramel candy, a water-soluble material, as the adhesive for placement of brackets on the model. This technique was further modified by Moskowitz who proposed the usage of a thermally cured composite. Moin and Dugon used drops of sticky wax and polyether material for bracket placement and tray transfer. ${ }^{3}$ Thomas suggested the use of composite resin for sticking brackets on cast and thermoplastic sheet for tray transfer. ${ }^{4}$ Fried and Newman suggested the use of soluble wallpaper paste as a no-mix adhesive for the attachment of brackets on the cast. ${ }^{5}$

Hickman ${ }^{6}$ in 1993 elaborated a "dual-tray" transfer system for a chemically cured composite technique developed by Nakaji and Sheffield. ${ }^{7}$ However, Cooper and Sorenson ${ }^{8}$ in 1993, Kalange ${ }^{9} 1999$, and Sondhi ${ }^{10} 1999$ developed the concept of adhesive precoated brackets. Sinha et al. ${ }^{11}$ advocated the use of thermally cured, fluoride-releasing indirect bonding system. White ${ }^{12}$ suggested the use of a combination of tacky and hot glue. However, Vashi and $\mathrm{Vashi}^{13}$ suggested the addition of impression compounds along with tacky glue to increase the rigidity of the transfer tray. Bhardwaj et al. ${ }^{14}$ used a double-sided sticky tape to place brackets on the working cast and then used a soft transfer tray made up of a vacuum-formed thermoplastic material.

Read and $O^{\prime} B$ Brien ${ }^{15}$ and Read and Pearson ${ }^{16}$ suggested the use of a transparent thermoplastic sheet for tray transfer for making the indirect bonding technique compatible for light cure composite. Aileni et al. ${ }^{17}$ used Erkogum block-out wax as an adhesive to attach the brackets onto the cast and a thermoplastic glue was used to fabricate the transfer trays. Madhusudhan et al. ${ }^{18}$ and Rajagopal et al. ${ }^{19}$ used a micropore adhesive tape with
${ }^{1}$ KM Shah Dental College and Hospital, Vadodara, Gujarat, India

${ }^{2,3}$ Department of Orthodontics and Dentofacial Orthopaedics, KM Shah Dental College and Hospital, Vadodara, Gujarat, India

Corresponding Author: Shrishtee Manoj Goyal, Department of Orthodontics and Dentofacial Orthopaedics, KM Shah Dental College and Hospital, Vadodara, Gujarat, India, Phone: +91 9893985437, e-mail: shrisgoyal@gmail.com

How to cite this article: Kulkarni NB, Goyal SM, Shah VD. 3D Printing Pen: A Novel Adjunct for Indirect Bonding. J Contemp Dent Pract 2021;22(8):964-968.

Source of support: Nil

Conflict of interest: None

cyanoacrylate glue to attach the brackets to the cast and gelatin jigs were prepared over brackets for additional retention. Transfer trays were fabricated using a 2- $\mathrm{mm}$ thick bioplast.

One of the drawbacks of indirect bonding is the incomplete curing of the composite ${ }^{20}$ due to partial light penetration through the transfer tray, leading to a weaker bracket bond. Other drawbacks include additional laboratory time, ${ }^{21}$ the need for an additional set of impressions, and technique sensitivity. ${ }^{4}$

Three-dimensional printing is a process that builds solid objects through the incremental addition of layers. The material used for 3D printing is polylactic acid (PLA). A 3D printing pen is a device that uses a PLA filament and softens it to construct solid 3D objects. PLA is a thermoplastic polymer with a melting range of 170 to $210^{\circ} \mathrm{C}$. It is a biodegradable polymer that has recently gained popularity in the field of medicine as it has been known to show no harmful effects on the human body. ${ }^{22}$

PLA is apt for the fabrication of the transfer tray for indirect bonding since it is rigid and transparent. It can be fabricated for an individual tooth or a group of teeth as per the requirement of the operator and anatomical constraints (undercuts, additional 
cusp tip, etc.) The literature suggested the need for improvement in the technique employed with the advent of newer biomaterials. Hence, a new indirect bonding technique by modifying the design and material of the transfer tray was attempted for better light penetration and bracket positioning accuracy.

\section{TECHNIQUe}

Five patients indicated for orthodontic treatment were selected from the OPD for the assessment of the new technique. Adequate information about the technique was explained to all patients, and the technique was employed to only those patients who were willing to participate. Informed consent was obtained from all participants. Bracket failure was assessed for a period of 12 months at a regular appointment interval of 4 weeks.

\section{Preparation of Transfer Tray}

Alginate impressions of upper and lower arches were made and casts were poured (Orthokal). The casts were retrieved and vertical and horizontal markings were drawn for bracket positioning according to the MBT bracket positioning chart ${ }^{23}$ with a lead pencil. A layer of bonding agent (3M Unitek) was applied over the surface of the cast and light-cured (Figs $1 \mathrm{~A}$ and B). The brackets (D-Tech, 0.022" slot) were fixed on the working cast by applying a layer of bonding agent on the bracket base and subjected to light-curing (Figs $1 C$ and D). The 3D-printed transfer trays made up of PLA (MatterHackers PRO PLA) were manually fabricated with the help of a 3D printing pen (3Doodler PRO) (Figs 1E and F). The pen was set at maximum flow and $210^{\circ} \mathrm{C}$ to ensure easy and adequate flow of the molten material. The tip of the 3D printing pen was held in proximity to the brackets; molten PLA was flown in such a way that three margins of the bracket, i.e., mesial, distal, and gingival, remained free of PLA; however, it engaged the slot of the bracket and extended on the occlusal and palatal surfaces of the tooth. Once the transfer tray was fabricated, the brackets were dislodged from the study model with the help of deboning pliers, maintaining the PLA tray intact (Figs $1 \mathrm{G}$ and $\mathrm{H}$ ). The remnants of plaster (if any) on the bracket base in the transfer tray were cleaned with the help of an intraoral sandblaster. The transfer tray was evaluated for proper fit on the teeth, following which the curing procedure was carried out.

\section{Preparation for Clinical Bonding}

Oral prophylaxis was performed to remove plaque and debris from the teeth surfaces and glycerine-free pumice was used to clean the dentition. The working area was isolated and the labial surfaces of the teeth to be bonded were etched using $37 \%$ phosphoric acid for 30 seconds and then rinsed and dried. ${ }^{17} \mathrm{~A}$ bonding agent was applied on the labial surfaces of the teeth and light-cured. Composite resin (3M Unitek) was applied on the bracket bases which were held in the $3 \mathrm{D}$-printed transfer tray; these were then placed over the primed teeth (Figs $2 \mathrm{~A}$ and $\mathrm{B}$ ) and flash was removed with the help of a scaler. Once the snug fit of the transfer tray was ensured, all teeth were light-cured to finish the bonding procedure (Figs $2 \mathrm{C}$ and D). The transfer tray was dislodged from the brackets using a straight probe (Figs $2 \mathrm{E}$ and F). The breakage of PLA in the bracket slot was removed using a heated probe. Any residual resin present on the incisal margin of the bracket was removed using a tungsten carbide bur for the completion of the bonding procedure (Fig. 3). The lower left central incisor was not included in the bonding procedure because of inadequate space.

A total of three bracket failures were observed among all participants included in the study.
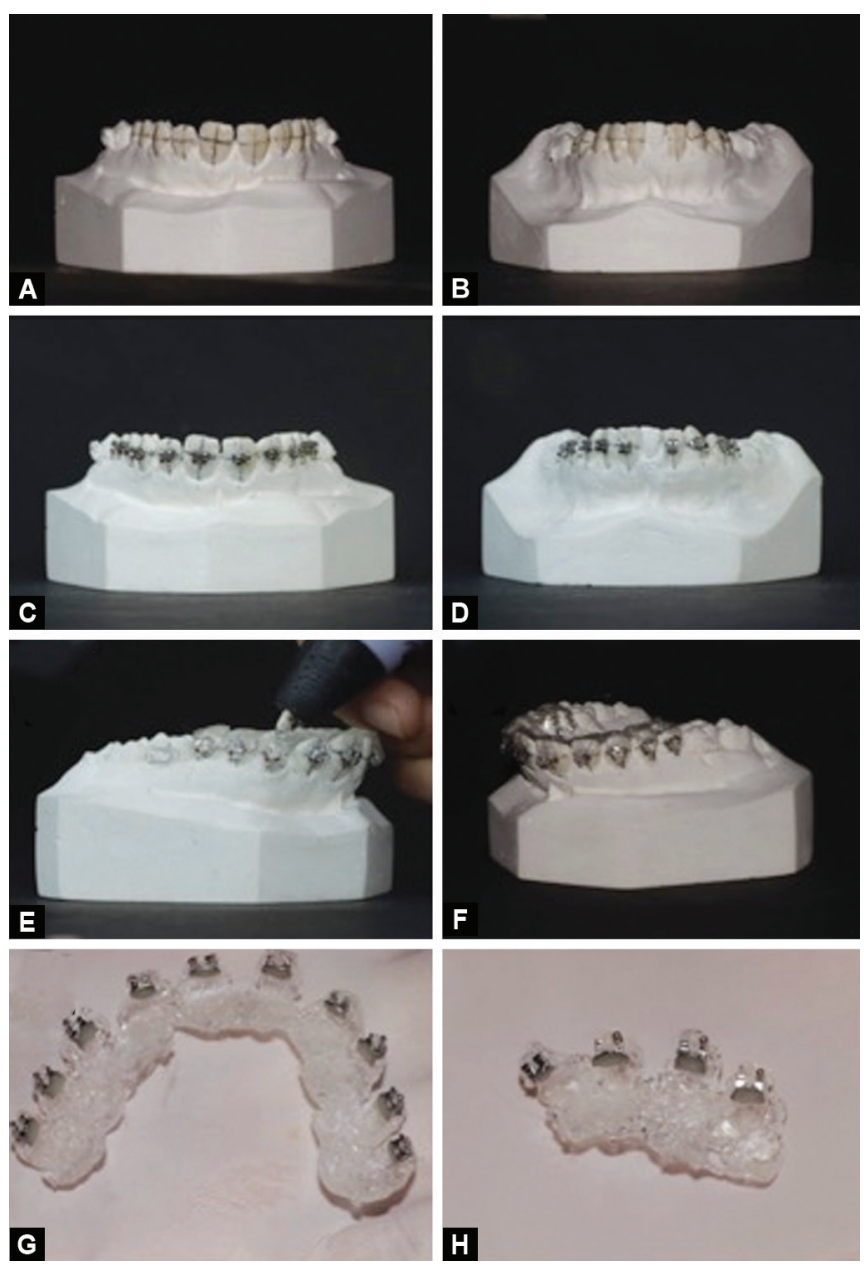

Figs $1 \mathrm{~A}$ to $\mathrm{H}$ : Laboratory procedures. (A) Markings for bracket positioning and layer of bonding agent applied on maxillary study model; (B) Markings for bracket positioning and layer of bonding agent applied on mandibular study model; (C) Brackets fixed on maxillary study model; (D) Brackets fixed on maxillary study model; (E) Fabrication of transfer tray; (F) Fabricated transfer tray; (G) Complete transfer tray; (H) Partial transfer tray

\section{Discussion}

Silverman et al. ${ }^{1}$ used a chemically cured composite for indirect bonding which gave the clinician less time for the clinical procedures. This drawback led to increased use of light-cured composite as the procedure of indirect bonding was refined over time. Read and O'Brien ${ }^{15}$ and Read and Pearson ${ }^{16}$ were the pioneers in the use of light cure composite for indirect bonding.

The first adhesive used to attach the brackets to the working model was bis-GMA, which was eventually changed to caramel candy by Shwartz; ${ }_{i}^{2}$ sticky wax by Moin and Dugon ${ }^{3}$ which added another cumbersome process of sticky wax removal from bracket base; composite resin by Kalange and Thomas ${ }^{4}$ which makes the procedure expensive; a double-sided tape by Bhardwaj et al. ${ }^{14}$ which required a precisely cut small dimension of the doublesided tape under the bracket base; Erkogum by Aileni et al. ${ }^{17}$ which provided sufficient time for bracket positioning on the cast but since it does not stiffen, the brackets may move from the desired position while the transfer tray is being fabricated; and micropore adhesive tape with cyanoacrylate glue by 

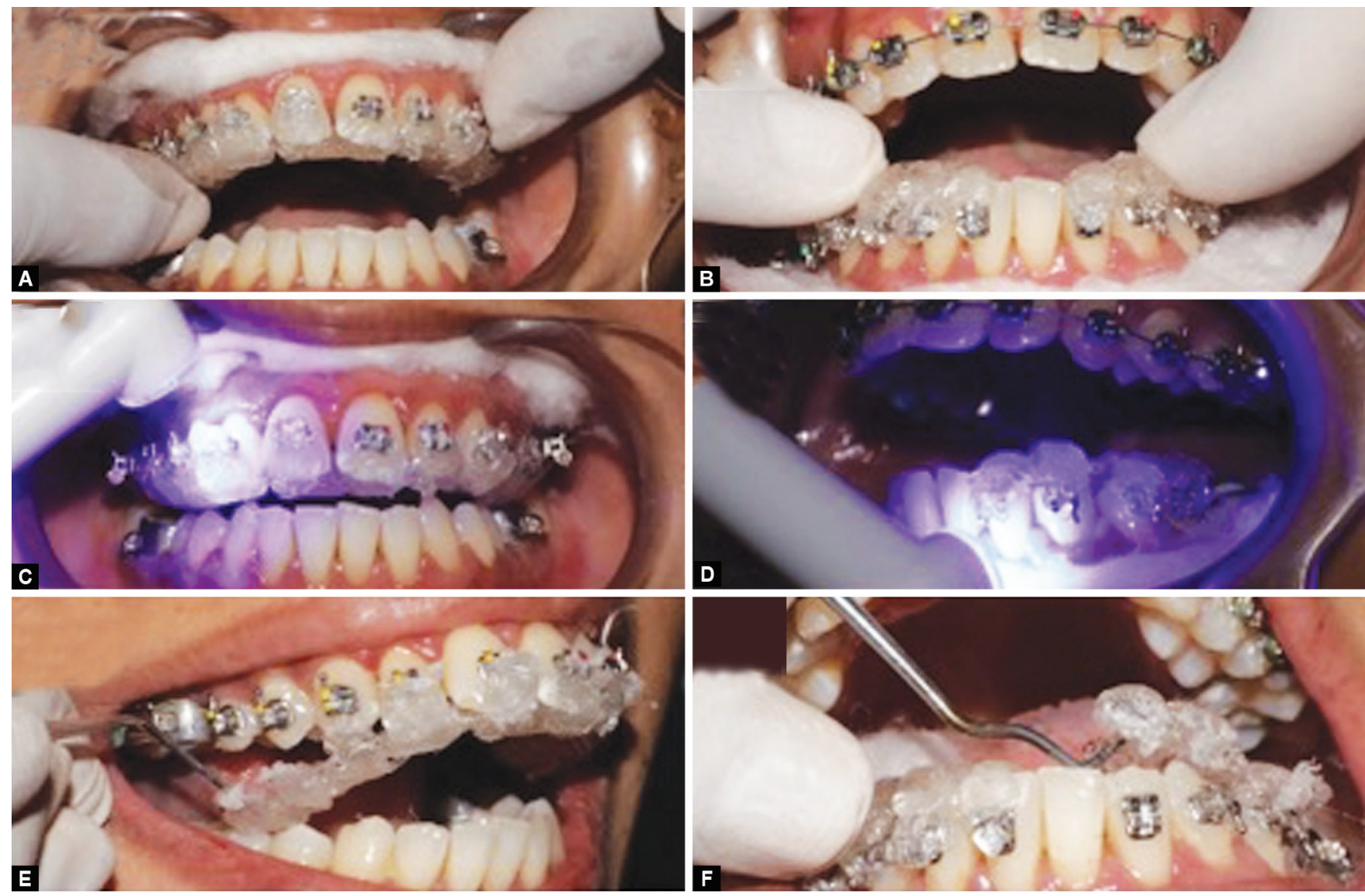

Figs 2A to F: Clinical procedures for indirect bonding. (A) Tray placement on maxillary dentition; (B) Tray placement on mandibular dentition; (C and D) Curing of composite; (E) Removal of the maxillary tray from the mouth; (F) Removal of the mandibular tray from the mouth

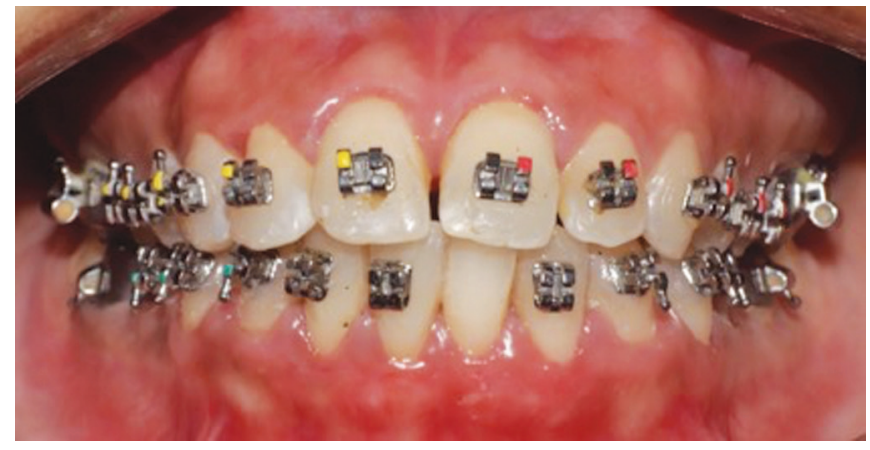

Fig. 3: Completed bonding procedure

Madhusudhan et al. ${ }^{18}$ which is known to be a potential health hazard. However, these studies exhibited no significant variation in results when different adhesives were used to attach brackets to the working models.

After reviewing the literature, we did not find any study that used the bonding agent as an adhesive to attach the brackets on the study models. We used a bonding agent as an adhesive to attach brackets to the study models in this case because it can be applied on the bracket base and a layer on the cast effortlessly. It also forms a very thin interface between the bracket base and the study model, thus increasing the accuracy in the expression of bracket prescription. Unlike Erkogum and sticky wax, the bonding agent is not affected by the heat of the molten PLA, and the bonding agent is highly biocompatible as compared to other materials used for indirect bonding.

Various materials have been used to fabricate the transfer tray starting with rubber-based impression materials by Moskowitz et al. ${ }^{24}$ to transparent vacuum-formed hard and soft thermoplastic sheets, ${ }^{6,7}$ thermoplastic glue, impression compounds, cold cure acrylic, and a combination of two materials. Some of these are made up of opaque materials, hence requiring a chemically cured composite. ${ }^{2}$ Even the transfer trays made up of transparent materials have the drawback of refraction of light ${ }^{25}$ leading to incomplete curing of the composite and thus bracket bond failure, digitally designed and 3D-printed PLA transfer trays are also used, but this method is not widely accepted due to the additional need for skills to design the tray over a software and expensive armamentarium like 3D scanner and printing machines, or it needs to be outsourced. Also, a study by Plattner et al. ${ }^{26}$ showed a higher overall production time for digitally produced indirect bonding trays as compared to laboratory-produced indirect bonding trays.

Other drawbacks include additional laboratory time, the need for an additional set of impressions, and technique sensitivity.

The PLA transfer tray is proved to be a handy tool for the indirect bonding procedure. When compared with the thermoformed vacuum-pressed polycarbonate sheets, fabrication of the PLA tray in the laboratory comparatively required more skills and took longer (5.8 minutes) than fabricating the vacuum-formed thermoplastic tray (3.4 minutes); however, the PLA tray fit snugly 
over the surfaces of the teeth and exhibited good rigidity when compared with the thermoformed polycarbonate trays.

The PLA transfer tray exhibited superior results compared with the thermoplastic tray for indirect bonding in terms of being more clinician-friendly and easy to fabricate and manipulate; it allowed for three free surfaces (mesial, distal, and gingival) for curing and for optimal bond strength. It had limited chairside corrections to be made, the brackets precisely and firmly adhered in the transfer tray, and the removal of the tray from the oral cavity was easier causing negligent bracket dislodgements.

When compared with other techniques of indirect bonding, the PLA transfer tray permitted the removal of flash from the bracket bases which is one of the prime factors for plaque accumulation.

The precision of placement of brackets is enhanced in the indirect bonding technique due to a two-step procedure (laboratory + clinical). Since the brackets are positioned outside the mouth on a study model, they can be viewed from various aspects, and under direct vision, the bracket positioning is more precise as compared to the direct bracket bonding procedure. The additional advantages include reduced chairside time and enhanced comfort for the patient and the clinician. Indirect bonding also provides comfort and ease when lingual orthodontic bonding procedures are performed. It is very cumbersome to carry out a direct bonding procedure on posterior teeth from premolars to molars; this technique improves the bonding of bracket and molar tube on posterior teeth. ${ }^{27}$

Bozelli et al. ${ }^{28}$ in a study concluded that total mean time needed for indirect bracket bonding (laboratory + clinical) was 38.92 minutes while that of direct bracket bonding (clinical) was 29.76 minutes. But the time taken for only the clinical steps in indirect bracket bonding was 12.68 minutes, which is significantly lower than that of direct bracket bonding.

Time taken for laboratory procedures using bonding agent, 3D printing pen, and PLA was 18.44 minutes, and that for clinical procedures was 11.86 minutes. The total time for the upper and lower complete bonding procedure came out to be 30.3 minutes. The limitations of this technique include technique sensitivity, excessive flow of PLA through the 3D printing pen, and fragments of PLA getting stuck in the bracket slot while tray removal, which were removed using a heated probe. However, a further study can be planned to evaluate the efficacy in the same pretext.

\section{Conclusion}

It can be concluded that using a bonding agent is an easy and effective way to attach brackets to the study models and it provides sufficient strength for further laboratory processes involved in indirect bonding. Fabrication of complete or partial transfer trays for indirect bonding using PLA with the help of a 3D printing pen is an easy and reliable method. Further studies are required to assess the bond strength, bracket failure rate, and accuracy of bracket positioning when a bonding agent is used as an adhesive and PLA is used to fabricate the transfer tray.

\section{Clinical Significance}

A $3 D$ printing pen and PLA were used to fabricate transfer trays for indirect bonding which provided us with advantages like ease of manipulation, immense control over the flow of materials and the parts of bracket and cast to be covered in PLA, cost effectiveness, and transparency. This method does not require any expensive equipment and the materials being used are easily portable. The only drawback with this technique was the problem with the dislodgment of the PLA transfer tray from the bracket slot when it flows toward the gingival edge. This problem can be tackled by using a heated explorer to dislodge the PLA stuck in the bracket slot.

\section{References}

1. Silverman $E$, Cohen $M$, Gianelly $A A$, et al. A universal direct bonding system for both metal and plastic brackets. Am J Orthod 1972;62(3):236-244. DOI: 10.1016/s0002-9416(72)90264-3.

2. Swartz M. About orthodontic bonding, an interview with Dr. Michael Swartz. The Ortho Cyber J 2003.

3. Moin K, Dogon IL. Indirect bonding of orthodontic attachments. Am J Orthod 1977;72(3):261-275. DOI: 10.1016/0002-9416(77)90212-3.

4. Kalange JT, Thomas RG. Indirect bonding: a comprehensive review of the literature. In: Seminars in orthodontics, vol. 13(1). WB Saunders; 2007. p. 3-10.

5. Fried KH, Newman GV. Indirect bonding with a no-mix adhesive. J Clin Orthod 1983;17:414-415.

6. Hickham JH. Predictable indirect bonding. J Clin Orthod 1993;27(4):215-218.

7. Nakaji, Sheffield. Table clinic. 1981 AAO annual meeting.

8. Cooper RB, Sorenson NA. Indirect bonding with adhesive precoated brackets. J Clin Orthod 1993;27(3):164-166.

9. Kalange JT. Ideal appliance placement with APC brackets and indirect bonding. J Clin Orthod 1999;33(9):516-526.

10. Sondhi A: Efficient and effective indirect bonding. Am J Orthod 1999;115(4):352-359. DOI: 10.1016/s0889-5406(99)70252-0.

11. Sinha PK, Nanda RS, Ghosh J. A thermal-cured, fluoride-releasing indirect bonding system. J Clin Orthod 1995;29(2):97-100.

12. White L. New and improved indirect bonding technique.J Clin Orthod 1999;33(1):17-23.

13. Vashi N, Vashi B. An improved indirect bonding tray and technique. J Indian Orthod Soc 2008;42:19-23. DOI: 10.1177/0974909820080105.

14. Bhardwaj A, Belludi A, Gupta A, et al. Indirect bonding technique - a simplified novel technique. J Asian Pacific Orthod Soc 2011;2(3).

15. Read MJ, O'Brien KD. A clinical trial of an indirect bonding technique with a visible light-cured adhesive. Am J Orthod Dentofacial Orthop 1990;98(3):259-262. DOI: 10.1016/S0889-5406(05)81603-8.

16. Read MJF, Pearson AL. A method for light-cured indirect bonding. J Clin Orthod 1998;32(8):502-503.

17. Aileni KR, Rachala MR, Mallikarjun V, et al. Gum and gun: a new indirect bonding technique. J Indian Orthod Soc 2012;46(4_suppl. 1):287-291. DOI: 10.5005/jp-journals-10021-1107.

18. Madhusudhan S, Laxmikanth SM, Shetty PC. A newly simplified indirect bonding technique. Indian J Dent Sci 2012;4(4):81-83.

19. Rajagopal R, Venkatesan A, Gnanashanmugham K, et al. A new indirect bonding technique. J Clin Orthod 2004;38(11):600-602.

20. Smith RT, Shivapuja PK. The evaluation of dual cement resins in orthodontic bonding. Am J Orthod Dentofacial Orthop 1993;103(5):448-451. DOI: 10.1016/S0889-5406(05)81795-0.

21. Aksakalli S, Demir A. Indirect bonding: a literature review. Eur J Gen Dent 2012;1(1):6. DOI: 10.4103/2278-9626.101344.

22. Van den Eynde M, Van Puyvelde P. 3D printing of poly (lactic acid). In: Industrial applications of poly (lactic acid). Cham: Springer; 2017. p. 139-158.

23. McLaughlin RP, Bennett JC. Bracket placement with the preadjusted appliance. J Clin Orthod 1995;29(5):302-311.

24. Moskowitz EM, Knight LD, Sheridan JJ, et al. A new look at indirect bonding. J Clin Orthod 1996;30(5):277-281.

25. Yu N, Genevet P, Kats MA, et al. Light propagation with phase discontinuities: generalized laws of reflection and refraction. Science 2011;334(6054):333-337. DOI: 10.1126/science.1210713.

26. Plattner J, Othman A, Arnold J, et al. Comparative study between the overall production time of digitally versus conventionally produced 
indirect orthodontic bonding trays. Turk J Orthod 2020;33(4):232. DOI: 10.5152/TurkJOrthod.2020.18079.

27. Aggarwal P, Aggarwal R. Indirect bonding procedures in orthodontics a review. J Dents Dent Med 2018;1(4):120. DOI: 10.31021/jddm. 20181120.
28. Bozelli JV, Bigliazzi R, Barbosa HA, et al. Comparative study on direct and indirect bracket bonding techniques regarding time length and bracket detachment. Dental Press J Orthod 2013;18(6):51-57. Available from: https://doi.org/10.1590/S2176-94512013000600009 